\title{
PLC Based Induction Motor Fault Detection \& Control
}

\author{
Jayesh Ravindra Bharambe ${ }^{1}$, Mahendra Gopal Kapale ${ }^{2}$, Mohd. Aslam Mohd. Salim ${ }^{3}$ \\ ${ }^{1,2,3}$ UG studetns B.Tech Electrical Engineering, Godavari college of Engineering, Jalgaon \\ ${ }^{1}$ kapalemahendra@gmail.com, ${ }^{2}$ kapalemahendra@gmail.com, ${ }^{3}$ aslammohd6799@gmail.com
}

Received on: 16 July,2021

Revised on: 02 September, 2021

Published on: 04 September, 2021

\begin{abstract}
The past two decades, the industry has investigated condition-based maintenance philosophies including new technologies developed to ascertain the condition of plant equipment during operation. Specifically, techniques have been developed, to monitor the condition of sensors and their associated instrument channels while the plant is operating. Historically, process instrumentation channels have been manually calibrated at each refueling outage. This strategy is not optimal as the sensor conditions are checked periodically; therefore, faulty sensors can operate unnoticed for periods up to the calibration frequency if not detected by other surveillance tasks. Additionally, periodic maintenance strategies cause the unnecessary calibration of instruments that are operating correctly which can result in premature aging, damaged equipment, plant down time, and improper calibration under non-service conditions. In fact, recent studies have shown that less than $5 \%$ of process instrumentation being manually calibrated requires any correction at all. Therefore, plants are interested in monitoring sensor performance during operation and calibrating the sensors off-line whose condition warrants it. Input circuits consist of fire sensor. It serves as input to the fault detection system, $R Y B$ detection system.
\end{abstract}

Keywords: PLC, Induction motor, Fault detection

\section{I -INTRODUCTION}

$\mathbf{T}_{\mathrm{h}}$ he developments in the technology allows the motors used in industrial manufacturing processes to be monitored and controlled in real time. The monitoring and control of the motors provides higher efficiency and opportunity to foresee the faults and malfunctions. Asynchronous motors used in electromechanical systems ought to be monitored and controlled consistently during the manufacturing process because of the fact that the product quality can easily be affected by a malfunction of the motor. Various control methods are developed for three phase asynchronous motors in recent years. Those developments aim for fast, reliable and efficient manufacturing process. The traditional control methods are being substituted by modern and smart control systems [1-2]. The control of electric motors, especially asynchronous motors with their advantages and design compatibility, is important [3]. The optimum control of asynchronous motors can be achieved by frequency converters with high 


\section{International Journal of Innovations in Engineering and Science, www.ijies.net}

performance [4]. The control of asynchronous motors depend on many different parameters so that it is relatively more difficult than the control of DC motors [1]. Although there are several control methods for asynchronous motors, the use of speed drivers is common nowadays because of their relatively easiness. Additionally, many of the parameters that cannot be controlled with traditional control methods can be monitored and controlled with speed drivers.

\section{II -LITERATURE SURVEY}

Increasing demands on reliability and safety of technical plants require early detection of process faults. Methods are developed that enable earlier detection of process faults than conventional limit and trend checking based on single process variable. These methods encompass information from not just one process variable but also include non-measurable variables as process state, parameters and characteristics quantities, [1]. Some methods require accurate process models while others rely primarily on available historical process data. In this brief review paper is outlined introduction to the field with major methods and literature references.

A. Data Methods and Signal Models :-

- Limit checking and trend checking

- Data analysis (PCA)

- Spectrum analysis and parametric models

- Pattern recognition (neural nets)

B. Process Model Based Methods :-

- Parity equations

- State observers

- Parameter estimation

- Nonlinear models (neural nets)

C. Knowledge Based Methods :-

- Expert systems

- Fuzzy logic

System development:

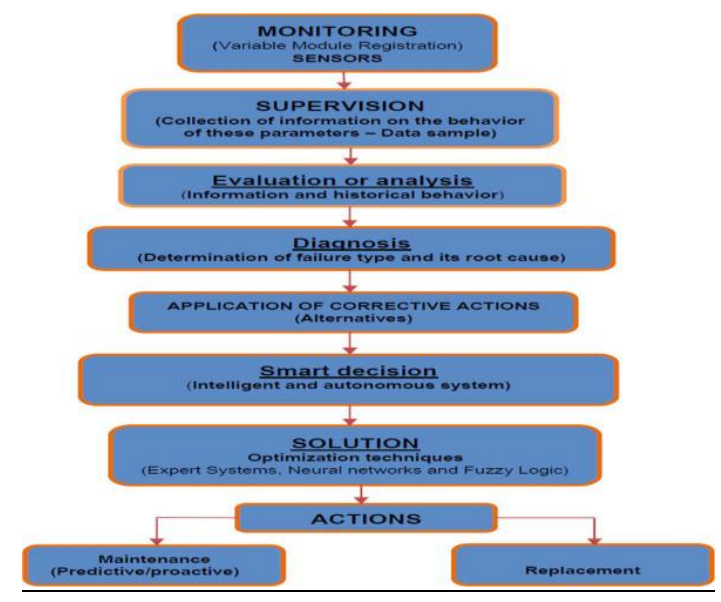

Fig.1- Algorithm-Software development

Major Types of Industrial Control Systems Industrial control system or ICS comprise of different types of control systems that are currently in operation in various industries. These control systems include PLC, SCADA and DCS and various others:

\section{PLC :-}

They are based on the Boolean logic operations whereas some models use timers and some have continuous control. These devices are computer based and are used to control various process and equipments within a facility. PLCs control the components in the DCS and SCADA systems but they are primary components in smaller control configurations.

$\underline{\text { DCS }}$

Distributed Control Systems consists of decentralized elements and all the processes are controlled by these elements. Human interaction is minimized so the labor costs and injuries can be reduced

\section{Embedded Control}

In this control system, small components are attached to the industrial computer system with the help of a network and control is exercised

\section{SCADA}

Supervisory Control And Data Acquisition refers to a centralized system and this system is composed of various subsystems like Remote Telemetry Units, Human Machine Interface, Programmable Logic Controller or PLC and Communications. Hardware Components of a PLC System processor unit (CPU), 


\section{International Journal of Innovations in Engineering and Science, www.ijies.net}

Memory, Input/Output, Power supply unit, Programming device, and other devices.

Hardware Processor unit (CPU), Memory, Input/Output, Power supply unit, Programming device and other devices.

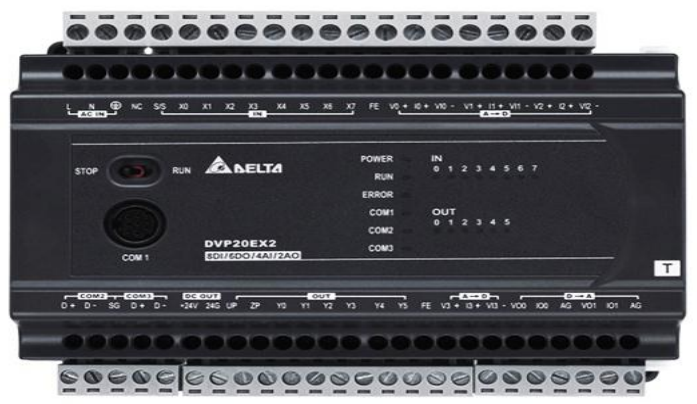

Fig. 2- PLC System processor unit (CPU)

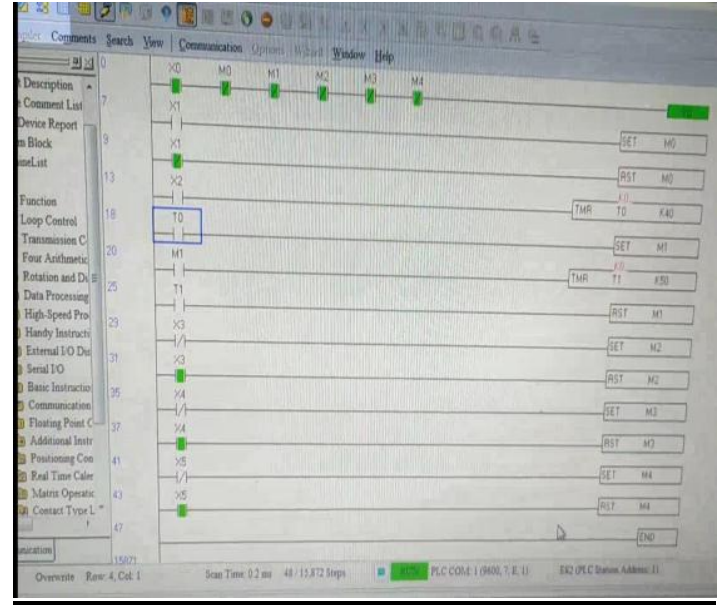

Fig. 3 -Wiring

\section{Central Processing Unit (CPU)}

CPU - Microprocessor based, may allow arithmetic operations, logic operators, block memory moves, computer interface, local area network, functions, etc. CPU makes a great number of check-ups of the PLC controller itself so eventual errors would be discovered early.System Busses The internal paths along which the digital signals flow within the PLC are called busses.
The system has four busses:

- The CPU uses the data bus for sending data between the different elements,

- The address bus to send the addresses of locations for accessing stored data, - The control bus for signals relating to internal control actions,

- The system bus is used for communications between the I/O ports and the I/O unit.

\section{Memory:-}

System (ROM) to give permanent storage for the operating system and the fixed data used by the CPU.RAM for data this is where information is stored on the status of input and output devices and the values of timers and counters and other internal devices. EPROM for ROM's that can be programmed and then the program made permanent.

\section{I/O Sections}

Inputs monitor field devices, such as switches and sensors. Outputs control other devices, such as motors, pumps, solenoid valves, and lights.

\section{Power Supply:}

Most PLC controllers work either at 24 VDC or 220 VAC. Some PLC controllers have electrical supply as a separate module, while small and medium series already contain the supply module. The programming device is used to enter the required program into the memory of the processor. The program is developed in the programming device and then transferred to the memory unit of the PLC.

\section{Input Relays :}

These are connected to the outside world. They physically exist and receive signals from switches, sensors, etc. Typically they are not relays but rather they are transistors.

\section{Internal Utility Relays :}




\section{International Journal of Innovations in Engineering and Science, www.ijies.net}

These do not receive signals from the outside world nor do they physically exist. They are simulated relays and are what enables a PLC to eliminate external relays. There are also some special relays that are dedicated to performing only one task.

\section{Counters:}

These do not physically exist. They are simulated counters and they can be programmed to count pulses. Typically these counters can count up, down or both up and down. Since they are simulated they are limited in their counting speed. Some manufacturers also include high speed counters that are hardware based.

\section{Timers:}

These also do not physically exist. They come in many varieties and increments. The most common type is an on-delay type. Others include off-delay and both retentive and non-retentive types. Increments vary from $1 \mathrm{~ms}$ through $1 \mathrm{~s}$.

\section{Output Relays :-}

These are connected to the outside world. They physically exist and send on/off signals to solenoids,

lights, etc.They can be transistors, relays, or triacs depending upon the model chosen.

\section{Data Storage :-}

Typically there are registers assigned to simply store data. Usually used as temporary storage for math or data manipulation. They can also typically be used to store data when power is removed from the PLC.

\section{Extension modules:-}

PLC I/O number can be increased through certain additional modules by system extension through extension lines. Each module can contain extension both of input and output lines. Extension modules can have inputs and outputs of a different nature from those on the PLC controller. When there are many I/O located considerable distances away from the PLC an economic solution is to use I/O modules and use cables to connect these, over the long distances, to the PLC.

\section{Remote I/O connections :}

When there are many I/O located considerable distances away from the PLC an economic solution is to use I/O modules and use cables to Connect these, over the long distances, to the PLC.

\section{Remote PLCs:}

In some situations a number of PLCs may be linked together with a master PLC unit sending and receiving $\mathrm{I} / \mathrm{O}$ data from the other units.

Cables :

Twisted-pair cabling, often routed through steel conduit. Coaxial cable enables higher data rates to be transmitted and does not require the shielding of steel conduit. Fiber-optic cabling has the advantage of resistance to noise, small size and flexibility.

\section{Parallel communication:}

Parallel communication is when all the constituent bits of a word are Simultaneously transmitted along parallel cables. This allows data to be transmitted over short distances at high speeds. Might be used when connecting laboratory instruments to the system.

\section{Parallel standards :}

The standard interface most commonly used for parallel communication is IEEE-488, and now termed as General Purpose Instrument Bus (GPIB).Parallel data communications can take place between listeners, talkers, and controllers. There are 24 lines: 8 data (bidirectional), 5 Status \& control, 3 handshaking, and 8 ground lines.

\section{Serial communication:}

Serial communication is when data is transmitted one bit at a time. A data word has to be separated into its constituent bits for transmission and then reassembled into the word when received. Serial communication is used for transmitting data over long distances. Might be used for the connection between a computer and a PLC.

\section{Serial standards :}

RS-232 communications is the most popular method of plc to external device communications. RS 232 is a communication interface included Under SCADA 


\section{International Journal of Innovations in Engineering and Science, www.ijies.net}

applications. Other standards such as RS422 and RS423 are similar to RS232 although they permit higher transmission rates and longer cable distances.

There are 2 types of RS-232 devices:

DTE - Data Terminal Equipment and a common example is a computer.

DCE - Data Communications Equipment and a common example is a modem.

PLC may be either a DTE or DCE device.

\section{ASCII:}

ASCII is a human-readable to computer-readable translation code (Each letter/number is translated to 1 's and 0 's). It's a 7-bit code, so we can translate 128 characters $\left(2^{\wedge} 7\right.$ is 128$)$.

\section{Protocols :}

It is necessary to exercise control of the flow of data between two devices so what constitutes the message, and how the communication is to be initiated and terminated, is defined. This is termed the protocol. One device needs to indicate to the other to start or stop sending data. Interconnecting several devices can present problems because of compatibility problems. In order to facilitate communications

between different devices the International Standard Organization (ISO) in 1979 devised a model to be used for standardization for Open System Interconnection (OSI).

\section{PERFORMANCE}

performance is boosted by optimizing power and improving the fault detection system to maximize efficiency.in our project our PLC is programmable by real time monitoring to detect and protect the motor from faulty conditions like are as follows:

\section{*TempratureRise \\ *RotorJamming \\ *phasefault.etc,}

if we use PLC system our efficiency is boosted by 60 to $70 \%$ and time wastage is vary less because of the milliseconds operation a fault detection and protection system is developed for the driver parameters of three phase asynchronous motors by using PLC. As the fundamental units in the developed system, IM motor, PLC (Programmable Logic Controller) is used. The basis of the fault detection and protection mode is generated by comparing the parameters received from the driver by the software with the optimum parameters. As a result of the study, it is confirmed that the controlling of the parameters belonging to the asynchronous motor can be fast and reliable.

For the past two decades, the industry has investigated condition-based maintenance philosophies including new technologies developed to ascertain the condition of plant equipment during operation. Specifically, techniques have been developed, to monitor the condition of sensors and their associated instrument channels while the plant is operating. Historically, process instrumentation channels have been manually calibrated at each refueling outage. This strategy is not optimal as the sensor conditions are checked periodically; therefore, faulty sensors can operate unnoticed for periods up to the calibration frequency if not detected by other surveillance tasks.

Additionally, periodic maintenance strategies cause the unnecessary calibration of instruments that are operating correctly which can result in premature

aging, damaged equipment, plant down time, and improper calibration under non-service conditions. In fact, recent studies have shown that less than $5 \%$ of process instrumentation being manually calibrated requires any correction at all. Therefore, plants are interested in monitoring sensor performance during operation and calibrating the sensors off-line whose condition warrants it. Input circuits consist of temprature sensor, Motor jamming detection, 3 phase detection $(\mathrm{R}, \mathrm{Y}, \mathrm{B})$ also the output circuit will be provided with buzer and control based indications to indicate that fault has occurred in the system. This system will also facilitate continuous monitoring of the industrial plant.

For example 1] if because of some problem fire starts Buzzer and water motor will get on.

2] If load on motor get increases so CT will send information to PLC to make motor off. 
Vol. 6 , No. 10, 2021, PP. 232-237

\section{International Journal of Innovations in Engineering and Science, www.ijies.net}

\section{III -CONCLUSIONS}

The parameters of a 1 phase asynchronous motor are monitored and controlled successfully. Especially for the prevention of a motor fault in an industrial application, the developed system becomes important. The system can be used in experimental studies for investigations. Besides that, it can also be used for educational purposes in electric, electronics, mechanical, mechatronics and automation engineering fields. After the necessary modifications on the hardware and the software, the system will be able to monitor and control asynchronous motors with desired size and number. One of the important features of the system is the protection module. It is seen that the protection module enables the protection mode for the motor and send a warning message to the user when the system encounters a possible problem. The data regarding the working situations of the motor are recorded and displayed on the graphs. When compared to the existing systems, the developed system is an efficient, economic and compact system consisting of several properties.

\section{REFERENCES}

[1] Yllmaz C, Gürdal O, Koşalay I, "Network induced delay of asynchronous motor connectes to profibus-dp network using fuzzy logic control algorithm," Expert System with Applications, vol. 37, no. 32, pp. 3248-3255, 2010.

[2] Yuldiz, C, "Control of vectoral asynchronous motor by using genetic algorithm based fuzzy logic," MS Thesis, Kahramanmaras Sutcu Imam University, Institute of Natural Sciences, Turkey, 2008.

[3] $\ddot{O}, \quad C$, Abut, $N$, "The speed control of asynchronous motors by microprocessors," Elektrik Mühendisliği Dergisi, vol. 375, pp. 197202, 1990. [4] Jerkovic V, Spoljaric Z, Valter Z, "Optimal Control of Induction Motor Using High Performance Frequency Converter," IEEE, vol. 978, no. 1, pp. 1742-1744, 2008. 